\title{
Activity and tumor specificity of human heparanase gene core promoter
}

\author{
XIAO-PENG CHEN ${ }^{1}$, LIANG-HE HU ${ }^{2}$, WEI CUI ${ }^{1}$, DONG WANG ${ }^{1}$, \\ MING-SHI WU ${ }^{1}$, JUN-SHENG LUO ${ }^{1}$ and MIN ZHONG ${ }^{3}$ \\ ${ }^{1}$ Department of Hepatobiliary Surgery, Affiliated Yijishan Hospital of Wannan Medical College, Wuhu, Anhui 241001; \\ ${ }^{2}$ Department of General Surgery, Tongling People's Hospital, Tongling, Anhui 244000; \\ ${ }^{3}$ Central Laboratory, Affiliated Yijishan Hospital of Wannan Medical College, Wuhu, Anhui 241001, P.R. China
}

Received March 10, 2012; Accepted July 9, 2012

DOI: $10.3892 / \mathrm{mmr} .2012 .992$

\begin{abstract}
Heparanase (HPSE) plays a critical role in tumor metastasis and vascularization. In addition, the human HPSE promoter has been cloned and characterized. However, the activity and specificity of the HPSE promoter in tumor cells remains unclear. The core fragment of the HPSE promoter was amplified and cloned into the multiple cloning site of the pEGFP-1 vector. The recombinant plasmid pEGFP-Hp was transfected into human umbilical vein endothelial cells (ECV304) and human hepatoma carcinoma (HepG2), laryngocarcinoma (Hep2) and chronic myelogenous leukemia (K562) cell lines. The vectors pEGFP-1 and pEGFP-N1 were used as negative and positive controls, respectively. The activity and expression of green fluorescent protein (GFP) were analyzed. Results showed that the sequence of the amplified HPSE promoter was in agreement with the GenBank data. The recombinant plasmid pEGFP-Hp was consistent with the expected result. No GFP expression was observed in the transfected cells in the pEGFP-1 group, but a high expression was observed in the pEGFP-N1 group. As regards the pEGFP-Hp group, less fluorescence was revealed in ECV cells with a relatively high fluorescence in tumor cells. The average transfection efficiencies of pEGFP-Hp in the ECV304, HepG2, Hep2 and K562 cell lines were 3.9, $21.3,10.8$ and $6.5 \%$, respectively, while those of pEGFP-N1 were $17.1,24.0,14.0$ and $11.0 \%$, respectively. The HPSE gene promoter drives the expression of downstream genes in
\end{abstract}

Correspondence to: Dr Xiao-Peng Chen, Department of Hepatobiliary Surgery, Affiliated Yijishan Hospital of Wannan Medical College, No. 2 Zheshan West Road, Wuhu, Anhui 241001, P.R. China E-mail:drcxp@sohu.com

Abbreviations: ECV304, human umbilical vein endothelial cell line; HepG2, hepatoma carcinoma cell line; Hep2, laryngocarcinoma cell line; K562, leukemia cell line

Key words: heparanase, promoter, transcription factor binding site, gene therapy, vector a eukaryotic vector, specifically in tumor cell lines, but its activity is relatively weak.

\section{Introduction}

Heparanase (HPSE) is an endoglycosidase and plays a critical role in tumor metastasis and vascularization by specifically degrading the heparan sulfate chains of proteoglycan, a component of blood vessel walls and the extracellular matrix. Previous studies demonstrated that HPSE expression is increased in a variety of malignancies (1-8). The human HPSE gene and its genomic structure were cloned and characterized by several research teams simultaneously in 1999 (6-9). To understand the mechanisms of HPSE gene expression and regulation, the HPSE promoter sequence has been cloned and some transcription factor binding sites (TFBSs) have also been identified. Sequence analysis revealed that the TATA-less, GC-rich promoter of the HPSE gene belongs to the family of housekeeping genes. There were three Sp1, four Ets-relevant elements (ERE) and two early growth response gene-1 (EGR-1) binding sites within the core promoter area $(10,11)$. Sp1, GA-binding protein $(\mathrm{GABP})$ and EGR-1 were able to regulate HPSE promoter activity. HPSE has therefore become a new intensive research topic in the field of target gene therapy $(12,13)$.

Targeted cancer therapy requires a targeted, efficient and safe gene-transfer system. The application of a tumor-specific promoter is a basic method. The tumor-specific promoter plays a significant role in targeted gene therapy by regulating the specific expression of downstream therapeutic genes in tumor cells and reducing damage to normal tissues and cells (14-16). However, the effectiveness of available tumor-specific promoters is limited. Thus, it is necessary to investigate a new tumor-specific promoter and identify its activity and specificity.

Given that the HPSE promoter is also a tumor-specific promoter and plays a significant role in targeted gene therapy, it is crucial to understand its activity and specificity in tumor cells. In this study, we cloned the core fragment of the HPSE gene promoter, analyzed the TFBSs, constructed a tumor cell expression vector using the HPSE gene promoter and for the first time detected its transcriptional activity and specificity in diverse cell types. The present study is likely to provide an 
experimental basis for gene-targeted therapy using the HPSE promoter.

\section{Materials and methods}

Materials. Normal human umbilical vein endothelial (ECV304), human hepatocellular carcinoma (HepG2), laryngeal epithelial (Hep2), erythromyeloblastoid leukemia (K562), E. coli XL1-blue and BL21 cells were donated by Professor Xue-Long Wang from the Department of Parasitology of Anhui Medical University, China. The human genome extraction kit and PCR product purification kit were purchased from Tiangen Biotech Company (Beijing, China). The Taq enzyme, dNTP, cloning vector plasmid pMD18-T Simple, DNA Marker DL 2000 and DL15000 were purchased from Takara Biotechnology Company (Dalian, China). $\mathrm{T}_{4} \mathrm{DNA}$ ligase, XhoI and HindIII were purchased from MBI Fermentas (Burlington, ON, Canada). Tryptone, yeast extract and agar were purchased from Oxoid Company (Hampshire, UK). Lipofectamine 2000 plasmid kit and DMEM medium were purchased from Invitrogen Corporation (Carlsbad, CA, USA). Plasmid pEGFP-1 [multiple cloning site (MCS) not containing promoter sequences] and pEGFP-N1 [MCS containing cytomegalovirus promoter sequence] were purchased from Yili company (Hefei, China), and HPSE primers were synthesized by Sangon Biotech Company (Shanghai, China). The fluorescence microscope was purchased from Nikon (Tokyo, Japan), and the flow cytometer was from Beckman Coulter Inc. (Brea, CA, USA).

HPSE promoter PCR amplification and purification. The experiment was conducted with the informed consent of the volunteers and was approved by the Ethics Committee of our institutes (the Affiliated Yijishan Hospital of Wannan Medical College, Wuhu, Anhui and the Tongling People's Hospital, Anhui, China). Peripheral blood (20 $\mu$ l) was collected from healthy, normal volunteers. Genomic DNA was extracted according to the conventional method, and concentration and purity were determined by ultraviolet spectrophotometry. The primers were designed according to the human HPSE promoter Genbank sequence (GenBank Accession AF461265). The upstream primer (5'-GCTCAAGTGACAAGCAAGTGT TTAT-3') was synthesized using the only restriction enzyme cutting site (RECS) XhoI in the HPSE DNA sequence and no additional RECS was added. A HindIII restriction site (underlined) was introduced into the downstream primer (5'-CCA AGCTTGGGCTCACCTGGCTGCTC-3'). The core fragment of the HPSE gene promoter was amplified by PCR using $10 \mu \mathrm{l}$ genomic DNA as a template. The PCR conditions included initial denaturation at $95^{\circ} \mathrm{C}$ for $5 \mathrm{~min}, 35$ cycles of amplification with subsequent denaturation at $94^{\circ} \mathrm{C}$ for $55 \mathrm{sec}$, annealing at $57^{\circ} \mathrm{C}$ for $50 \mathrm{sec}$ and extension at $72^{\circ} \mathrm{C}$ for $50 \mathrm{sec}$, followed by a final extension at $72^{\circ} \mathrm{C}$ for $10 \mathrm{~min}$. Electrophoresis was performed using $1 \%$ agarose gel. The PCR product was purified according to the manufacturer's instructions and two-way sequencing was completed by Sangon Biotech Company.

Identification of the PCR products. XL1-blue competent cells were prepared using the calcium chloride method. PCR products and pMD18-T Simple were incubated overnight for ligation and then transformed into competent cells. Positive colonies were identified and amplified, plasmid DNA was extracted using the alkaline lysis method and identified by double digestion with XhoI and HindIII. Following 1\% agarose gel electrophoresis, $1 \mathrm{ml}$ bacterial suspension containing the recombinant plasmid was sent to Sangon Biotech Company for sequencing and the sequences were analyzed for putative TFBS.

Construction of recombinant plasmid vector $p E G F P-H p$. The recombinant plasmid was amplified and the DNA was extracted and digested by double digestion with XhoI and HindIII. The digested PCR products were analysed using agarose gel electrophoresis and then retrieved. The purified product was a DNA fragment of the HPSE promoter. The plasmid pEGFP-1 (4.2 kbp in length) was also digested by double digestion and the large fragment was retrieved. During a ligation reaction, both recycled products were incubated overnight at $4^{\circ} \mathrm{C}$ in order to insert the DNA fragment of the HPSE core promoter into the pEGFP-1 vector. After the competent cell suspension was thawed at room temperature, ligation products were added for transformation analysis. The recombinant plasmid was screened and the plasmid DNA was extracted by the alkaline lysis method, identified by double digestion, PCR amplification and DNA sequencing. The positive recombinant plasmid was designated as pEGFP-Hp.

Functional analysis of recombinant plasmid pEGFP-Hp. Cells were cultured in 96-well plates in RPMI-1640 cell culture medium with $10 \%$ fetal calf serum (FCS) and incubated at $37^{\circ} \mathrm{C}$ in a humidified atmosphere containing $5 \% \mathrm{CO}_{2}$. For each well, at $70-80 \%$ confluence, cells were inoculated into three wells at a density of $5 \times 10^{5}-1 \times 10^{6}$ cells/well and transfected with the pEGFP-Hp, pEGFP-1 and pEGFP-N1 plasmids. The procedure was performed according to the manufacturer's instructions for Lipofectamine 2000. The cells were subsequently cultured for $48 \mathrm{~h}$, the suspended cells were then collected. The adherent cells were digested by trypsin $(0.2 \%)$. The digestion was terminated by adding blood serum culture medium. The fluorescence intensity was observed using a fluorescence microscope and the percentage of light-emitting cells and transfection efficiency were determined by flow cytometry. All experiments were repeated at least three times.

\section{Results}

Amplification, identification and sequence analysis of HPSE core promoter. Electrophoresis of HPSE promoter PCR products revealed a specific band between 500 and $750 \mathrm{bp}$, which was consistent with the theoretical length of 561 bp (Fig. 1). After the PCR product was ligated with the pMD18-T Simple and transformed into competent cells, the plasmid was extracted. When the products were digested with $\mathrm{XhoI}$ and HindIII, two fragments were generated, 2,771 and $482 \mathrm{bp}$ in length. When the PCR product was digested with a single enzyme, only a 3,253-bp fragment was produced (Fig. 2).

The sequencing results were consistent with the GenBank database of human HPSE promoter nucleotide sequence (Accession AF461265's bl2seq). The 5' and 3' ends were located at 556 and 4 bp upstream of the transcription start site, respectively. With the downstream primer carrying restriction 


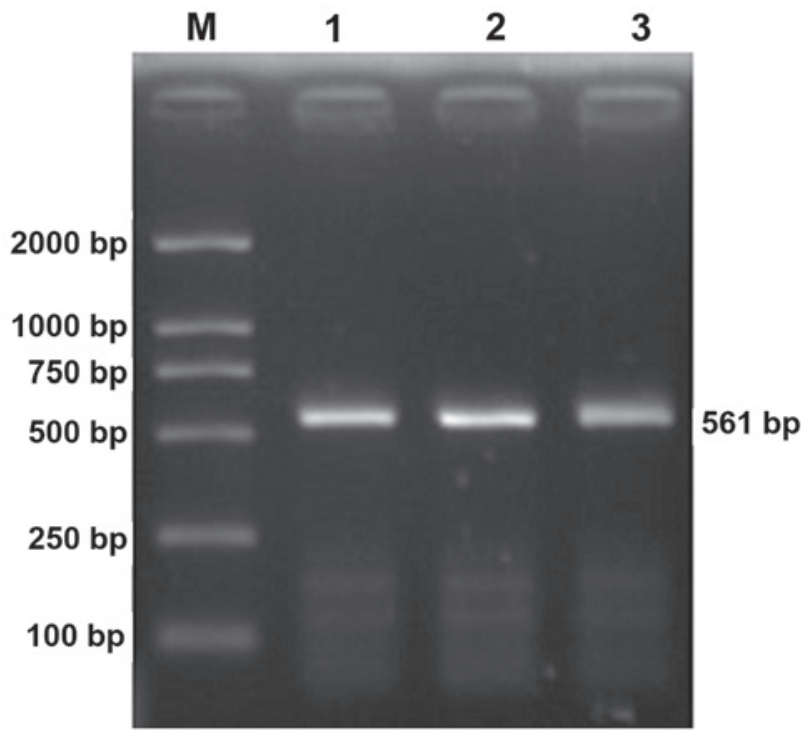

Figure 1. Electropherogram of HPSE PCR products revealed a specific band between 500 and $750 \mathrm{bp}$, which was consistent with the theoretical length of 561 bp. M, DL Marker 2000; lanes 1,2 and 3, PCR products. HPSE, heparanase.

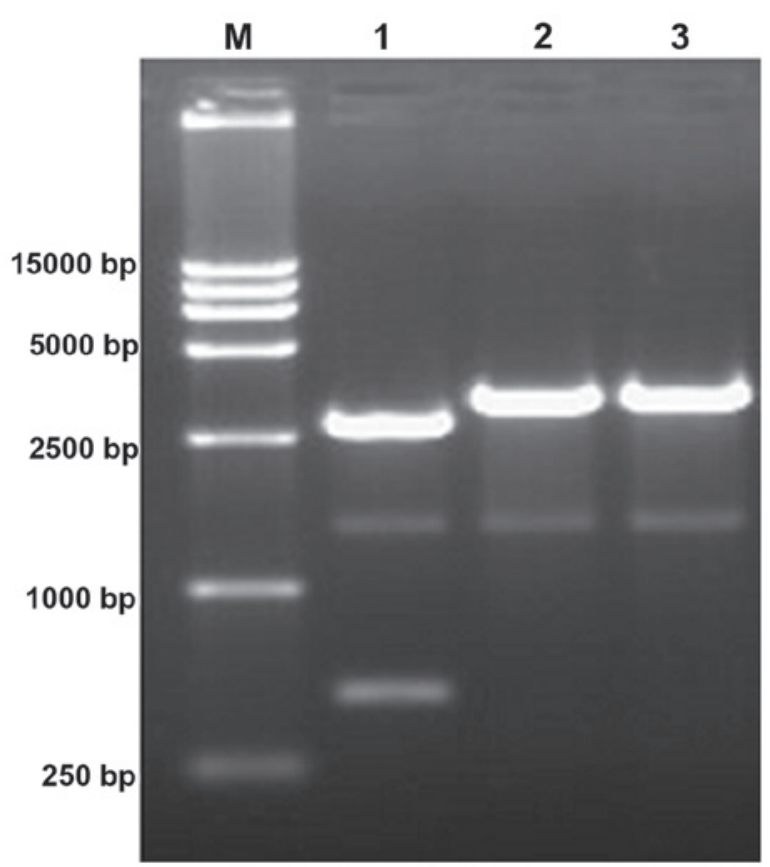

Figure 2. Electropherogram of the recombinant cloning vector digested by XhoI and/or HindIII revealed that two fragments were generated, 2,771 and 482 bp in length. M, DL Marker 15,000; lanes: 1, XhoI and HindIII double digest; 2 and 3, XhoI or HindIII single digest.

sites CCAAGCTT ( 8 bp), the total length was 561 bp. Sequence analysis revealed that the fragment contained the following TFBSs, as reported in previous studies $(8,9)$ : three $\mathrm{Sp} 1$, four ERE, two EGR-1, one E47, one N-myc and one NGFIp300. In addition, there are six potential E-box sites located at 553-548, 542-537, 402-397, 286-281, 34-29 and 14-9 bp upstream of the transcription start site (Fig. 3).

Identification of plasmid pEGFP-Hp. Two fragments were identified following electrophoresis when the recombinant plasmid pEGFP-Hp was subjected to double digestion with XhoI and HindIII. Only a single fragment was observed when the plasmid was digested by XhoI or HindIII. This proves that the recombinant plasmid was correctly constructed (Fig. 4). When plasmid pEGFP-Hp was used as a template, the HPSE promoter fragment was amplified, and the sequencing results were consistent with the GenBank data.

The activity and specificity of HPSE promoter. After cells were transfected with three types of plasmids, the fluorescence analysis showed that there was no fluorescence in any of the cells from the pEGFP-1 group, but a strong fluorescence in all the cells from the pEGFP-N1 group. In the pEGFP-Hp group, almost no fluorescence was detected in ECV304 cells, a degree of fluorescence was observed in all cancer cells other than ECV304 cancer cells, a strong expression was observed in the HepG2 and Hep2 cells and a relatively weak fluorescence was evident in K562 cells (Fig. 5). Flow cytometric analysis showed that the average transfecion efficiencies of pEGFP-Hp were $3.9 \pm 0.3,21.3 \pm 2.1,10.8 \pm 1.1$ and $6.5 \pm 0.6 \%$ in ECV304, HepG2, Hep2 and K562 cells, respectively. The transfection efficiencies of pEGFP-Nl were 17.1 $\pm 1.7,24.0 \pm 2.5,14.0 \pm 1.3$ and $11.0 \pm 1.0 \%$, respectively, which were all notably higher than those of pEGFP-Hp. The ratios of transfection efficiency of the two vectors in ECV304, HepG2, Hep2 and K562 cells were $0.23,0.89,0.77$ and 0.59 , respectively; all $<1$.

\section{Discussion}

The HPSE gene promoter is located in a region 2,300 bp upstream of the ATG translation initiation site. It lacks a TATA box but contains GC-rich regions. There were three Sp1, four ERE and two EGR-1 binding sites within the core promoter area $(9,10)$. In the present study, the HPSE promoter core fragment was cloned. Sequence analysis showed that it contained the above TFBSs, as described in the literature $(9,10)$. In addition, six E-box binding sites were identified, which were located at 553-548, 542-537, 402-397, 286-281, 34-29, 14-9 bp upstream of the ATG translation initiation site. These results suggest that the expression of heparanase gene is likely affected by the regulation of E-box sites.

One of the key factors of oncogene-targeted therapy is the selective or specific expression of a therapeutic gene in tumor cells. The application of tumor-specific promoters is one of the basic methods. A tumor-specific promoter drives downstream therapeutic genes that are only expressed in tumor cells, thereby reducing damage to normal tissue cells (14-16). At present, commonly used promoters include $\alpha$-fetoprotein (17), carcinoembryonic antigen (18), prostate-specific antigen (19), human telomerase reverse transcriptase (20) and multidrug resistance gene promoters (21). Tumor-specific promoters play a significant role in targeted gene therapy by combining with suicide gene, antioncogene, antisense nucleic acid, apoptosis gene and RNA interference.

Since HPSE mRNA and protein are expressed in the majority of tumor cells, but are not expressed in normal cells (1-8), the HPSE promoter may be used to construct a tumor-specific expression vector to guide the expression of a therapeutic gene. The vector may reduce or avoid adverse effects and improve the specificity and safety of gene therapy. 
GCTCAAGTGACAAGCA

E-box6

-540 AGTGTTTATAAGCTAGATGGGAGAGGAAGGGATGAATACTCCATTGGAGGTTTTACTCGA E-box5

-480 GGGTCAGAGGGATACCCGGCGCCATCAGATGGGATCTGGGAGTCGGAACGCTGGGTTC

-420 CCACGAGAGCGCGCAGAACACGTGCGTCAGGAAGCCTGGTCCGGGATGCCCAGCGCTGCT E-box4

-360 CCCCGGGCGCTCCTCCCCGGGCGCTCCTCCCCAGGCCTCCCGGGCGCTTGGATCCCGGCC

-300 ATCTCCGCACCCTTCAAGTGGGTTGGGTGATTTCGTAAGTGAACGTGACCGCCACCGAG

E-box3

-240 GGGAAAGCGAGCAAGGAAGTAGGAGAGAGCCGGGCAGGCGGGGCGGGGTTGGATTGGGAG

-180 CAGTGGGAGGGATGCAGAAGAGGAGTGGGAGGGATGGAGGGCGCAGTGGGAGGGGTGAGG

-120 AGGCGTAACGGGCGGAGGa aag agaaagggcgctggggctcggcgggaggaagtgct

$-60 \quad$ Agagctctcgactctccgctgcgcggagctggcgggggagcagcaggtgagccaag

E-box2

E-box1

$+1 \quad A T G \mathrm{ct}$

Figure 3. E-box TFBSs in cloned human heparanase gene core promoter.

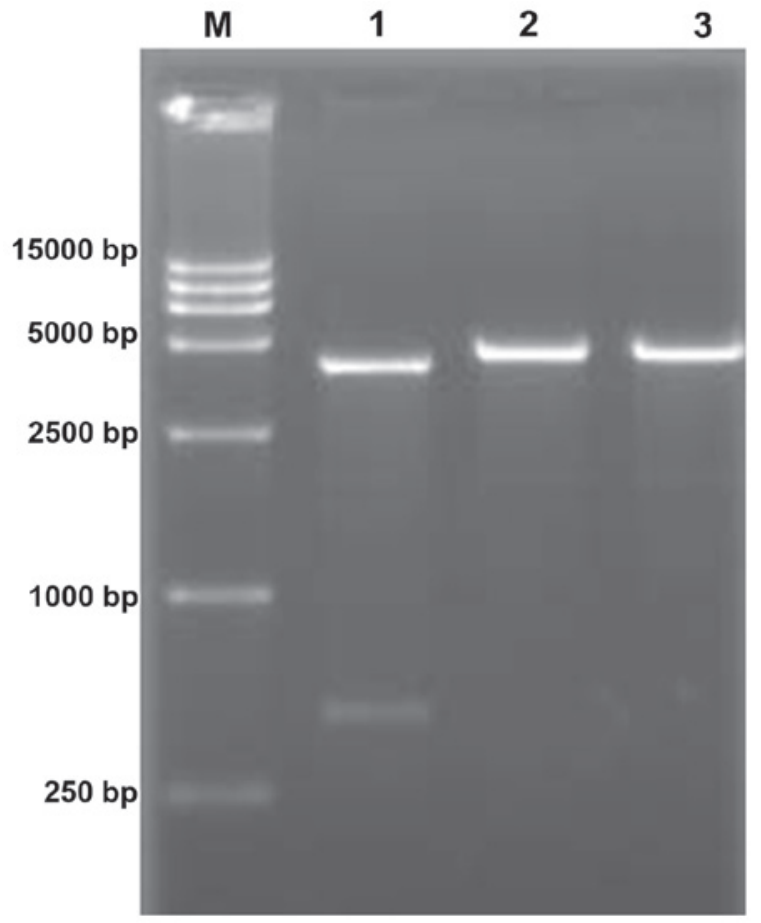

Figure 4. Electropherogram of recombinant plasmid pEGFP-Hp digested by XhoI and HindIIII. A single fragment was observed when digested by XhoI or HindIII, confirming the accuracy of the construction of the recombinant plasmid. M, DL Marker 15,000; lanes: 1, XhoI and HindIII; 2 and 3, XhoI or HindIII.

In this study, for the first time, the HPSE promoter fragment was inserted into the MCS of pEGFP-1 to construct the HPSE promoter-driven eukaryotic expression vector pEGFP-Hp, in which the HPSE promoter regulates the transcription and expression of the downstream GFP gene. After the recombinant vectors were transfected into normal and tumor cells, fluorescence observation and flow cytometric analysis showed
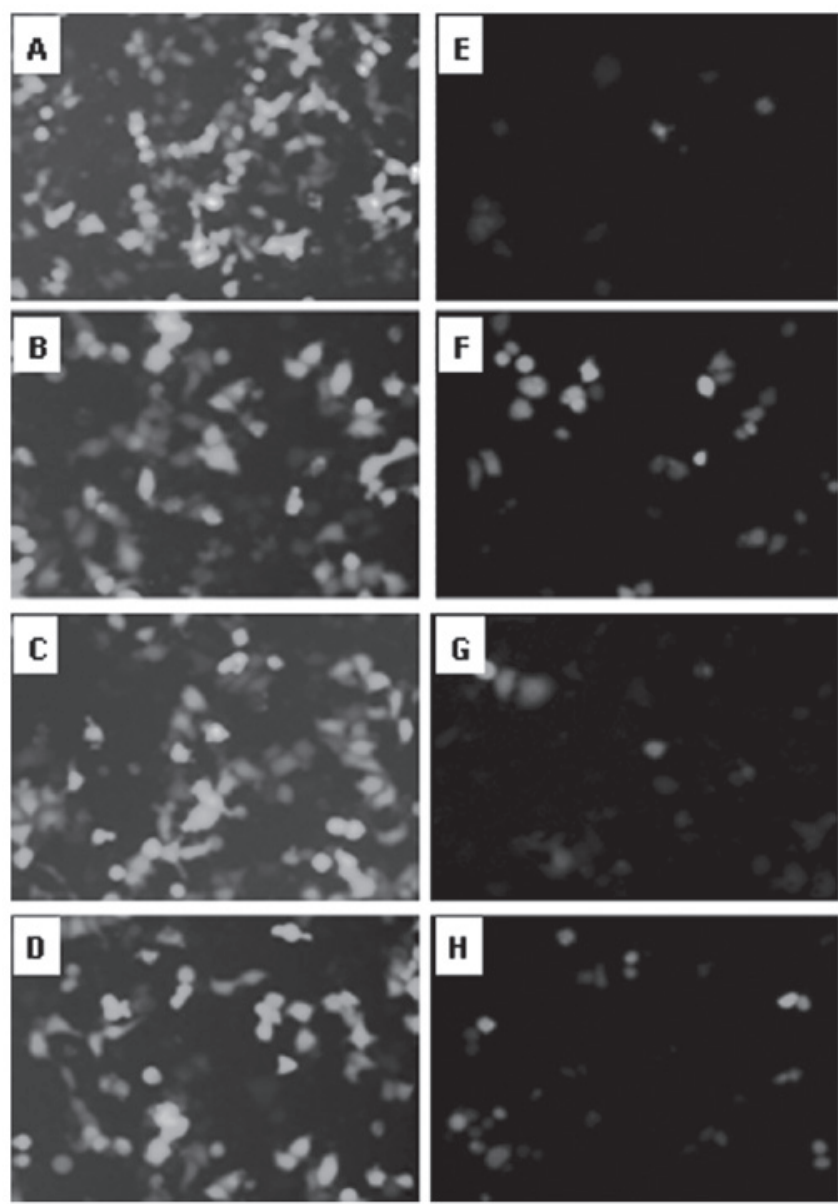

Figure 5. Fluorescence expression of pEGFP-N1 and pEGFP-Hp in various types of cells. (A-D) pEGFP-N1 in ECV304, HepG2, Hep2 and K562 cells, respectively. (E-H) pEGFP-Hp in ECV304, HepG2, Hep2 and K562 cells, respectively. Plamid pEGFP-N1 showed a strong fluorescence expression in normal and tumor cells. The recombinant plasmid pEGFP-Hp showed less fluorescence expression in normal cells and median-level expression in tumor cells, which was weaker than that of pEGFP-N1. 
that pEGFP-Hp does not initiate the transcription of the downstream GFP gene in normal cells. However, pEGFPHp drove GFP expression in tumor cell lines, which was consistent with the HPSE mRNA and/or protein expression levels in these cells (1-8). Since the transcriptional activity of pEGFP-N1 was strong in all the cells and pEGFP-1 demonstrated no pEGFP-N1 activity, this suggests that the HPSE promoter within the recombinant vector $\mathrm{pEGFP-Hp}$ regulates the tumor cell-specific expression of downstream genes. The HPSE promoter was therefore correlated with HPSE mRNA and/or the protein expression level. These findings are likely to provide new methods and an experimental basis for future targeted therapy for tumor-specific genes. Further experimentation is necessary to determine whether the HPSE promoter was tumor-specific.

In the same experimental conditions, the activity of the promoter in the expression vector determines its transfection efficiency in cells. Transfection efficiency, in turn, reflects the promoter activity. In this study, the transfection efficiencies of pEGFP-Hp in the four cell types were all lower than that of pEGFP-N1. This demonstrates that HPSE promoter activity is weak. Therefore, the efficiency of the HPSE promoter to drive therapeutic gene expression in tumor cells may not be as effective as we expect. Consequently, it exhibits drawbacks as an ideal gene therapy. Thus, future investigations should be performed to appropriately modify the HPSE promoter and increase its activity.

In summary, we successfully amplified the core fragment of the HPSE promoter and constructed the recombinant plasmid pEGFP-Hp by inserting it into the plasmid pEGFP-1. Following cell transfection using pEGFP-Hp, some degree of GFP expression was observed in tumor cells and no expression was identified in normal cells. The GFP expression level in tumor cells was weaker than that in the plasmid pEGFP-N1. These results demonstrate that the HPSE gene core promoter drives the vector expression specifically in tumor cell lines, but that its activity is relatively weak. The results may provide an experimental basis for tumor-targeting therapy using a tumorspecific promoter. Studies should be conducted to increase the activity of the HPSE promoter and evaluate whether the HPSE promoter was tumor-specific.

\section{Acknowledgements}

This study was supported by the Natural Science foundation of Anhui Province, China (Grant no. 11040606M181) and the Provincial Natural Science Foundation of College of Anhui Province, China (Grant no. kj2010B239). We would like to thank Professor Xue-Long Wang (Anhui Medical University, China) for technical assistance and Dr Li Gui (Anhui Medical University) for aid with flow cytometry.

\section{References}

1. El-Assal ON, Yamanoi A, Ono T, Kohno H and Nagasue N: The clinicopathological significance of heparanase and basic fibroblast growth factor expressions in hepatocellular carcinoma. Clin Cancer Res 7: 1299-1305, 2001.

2. Chen XP, Liu YB, Rui J, Peng SY, Peng CH, Zhou ZY, Shi LH, Shen HW and Xu B: Heparanase mRNA expression and point mutation in hepatocellular carcinoma. World J Gastroenterol 10: 2795-2799, 2004.
3. Komatsu N, Waki M, Sue M, Tokuda C, Kasaoka T, Nakajima M, Higashi $\mathrm{N}$ and Irimura T: Heparanase expression in B16 melanoma cells and peripheral blood neutrophils before and after extravasation detected by novel anti-mouse heparanase monoclonal antibodies. J Immunol Methods 331: 82-93, 2008.

4. Ilan N, Elkin M and Vlodavsky I: Regulation, function and clinical significance of heparanase in cancer metastasis and angiogenesis. Int J Biochem Cell Biol 38: 2018-2039, 2006.

5. Yingying X, Yong Z, Zhenning W, Xue Z, Li J, Yang L and Huimian X: Role of heparanase-1 in gastric carcinoma invasion. Asian Pac J Cancer Prev 10: 151-154, 2009.

6. Kussie PH, Hulmes JD, Ludwig DL, Patel S, Navarro EC, Seddon AP, Giorgio NA and Bohlen P: Cloning and functional expression of a human heparanase gene. Biochem Biophys Res Commun 261: 183-187, 1999.

7. Vlodavsky I, Friedmann Y, Elkin M, Aingorn H, Atzmon R, Ishai-Michaeli R, Bitan M, Pappo O, Peretz T, Michal I, et al: Mammalian heparanase: gene cloning, expression and function in tumor progression and metastasis. Nat Med 5: 793-802, 1999.

8. Toyoshima M and Nakajima M: Human heparanase. Purification, characterization, cloning and expression. J Biol Chem 274: 24153-24160, 1999.

9. Hulett MD, Freeman C, Hamdorf BJ, Baker RT, Harris MJ and Parish CR: Cloning of mammalian heparanase, an important enzyme in tumor invasion and metastasis. Nat Med 5: 803-809, 1999.

10. Jiang P, Kumar A, Parrillo JE, Dempsey LA, Platt JL, Prinz RA and $\mathrm{Xu} \mathrm{X}$ : Cloning and characterization of the human heparanase-1 (HPR1) gene promoter: role of GA-binding peotein and $\mathrm{Sp} 1$ in regulating HPR1 BASAL promoter activity. J Biol Chem 277: 8989-8998, 2002

11. de Mestre AM, Rao S, Hornby JR, Soe-Htwe T, Khachigian LM and Hulett MD: Early growth response gene 1 (EGR1) regulates heparanase gene transcription in tumor cells. J Biol Chem 280: 35136-35147, 2005

12. Boyd DD and Nakajima M: Involvement of heparanase in tumor metastases: A new target in cancer therapy? J Natl Cancer Inst 96: 1194-1195, 2004

13. Yang JM, Wang HJ, Du L, Han XM, Ye ZY, Fang Y, Tao HQ, Zhao ZS and Zhou YL: Screening and identification of novel B cell epitopes in human heparanase and their anti-invasion property for hepatocellular carcinoma. Cancer Immunol Immunother 58: 1387-1396, 2009.

14. Song MS, Jeong JS, Ban G, Lee JH, Won YS, Cho KS, Kim IH and Lee SW: Validation of tissue-specific promoter-driven tumor-targeting trans-splicing ribozyme system as a multifunctional cancer gene therapy device in vivo. Cancer Gene Ther 16: $113-125,2009$.

15. Fukazawa T, Maeda Y, Durbin ML, Nakai T, Matsuoka J, Tanaka H, Naomoto Y and Tanaka N: Pulmonary adenocarcinoma-targeted gene therapy by a cancer- and tissue-specific promoter system. Mol Cancer Ther 6: 244-252, 2007.

16. Huyn ST, Burton JB, Sato M, Carey M, Gambhir SS and Wu L: A potent, imaging adenoviral vector driven by the cancer-selective mucin-1 promoter that targets breast cancer metastasis. Clin Cancer Res 15: 3126-3134, 2009.

17. Willhauck MJ, Sharif Samani BR, Klutz K, Cengic N, Wolf I, Mohr L, Geissler M, Senekowitsch-Schmidtke R, Göke B, et al: Alpha-fetoprotein promoter-targeted sodium iodide symporter gene therapy of hepatocellular carcinoma. Gene Ther 15: 214-223, 2008.

18. Qiao J, Doubrovin M, Sauter BV, Huang Y, Guo ZS, Balatoni J, Akhurst T, Blasberg RG, Tjuvajev JG, Chen SH and Woo SL: Tumor-specific transcriptional targeting of suicide gene therapy. Gene Ther 9: 168-175, 2002.

19. Kraaij R, van der Weel L, de Ridder CM, van der Korput HA, Zweistra JL, van Rijswijk AL, Bangma CH and Trapman J: A small chimeric promoter for high prostate-specific transgene expression from adenoviral vectors. Prostate 67: 829-839, 2007.

20. Murofushi Y, Nagano S, Kamizono J, Takahashi T, Fujiwara H, Komiya S, Matsuishi T and Kosai K: Cell cycle-specific changes in hTERT promoter activity in normal and cancerous cells in adenoviral gene therapy: A promising implication of telomerasedependent targeted cancer gene therapy. Int J Oncol 29: 681-688, 2006.

21. Wang X, Ji C, Ma D, Zhao J, Hou M, Yu H and Zang S: Antitumor effects of cytosine deaminase and thymidine kinase fusion suicide gene under the control of mdrl promoter in mdr1 positive leukemia cells. Leuk Lymphoma 48: 1600-1609, 2007. 